\title{
OPEN The accuracy of several pose estimation methods for 3D joint centre localisation
}

\author{
Laurie Needham $\bowtie$, Murray Evans, Darren P. Cosker, Logan Wade, Polly M. McGuigan, \\ James L. Bilzon \& Steffi L. Colyer
}

Human movement researchers are often restricted to laboratory environments and data capture techniques that are time and/or resource intensive. Markerless pose estimation algorithms show great potential to facilitate large scale movement studies 'in the wild', i.e., outside of the constraints imposed by marker-based motion capture. However, the accuracy of such algorithms has not yet been fully evaluated. We computed 3D joint centre locations using several pre-trained deeplearning based pose estimation methods (OpenPose, AlphaPose, DeepLabCut) and compared to marker-based motion capture. Participants performed walking, running and jumping activities while marker-based motion capture data and multi-camera high speed images $(200 \mathrm{~Hz})$ were captured. The pose estimation algorithms were applied to 2D image data and 3D joint centre locations were reconstructed. Pose estimation derived joint centres demonstrated systematic differences at the hip and knee $(\sim 30-50 \mathrm{~mm})$, most likely due to mislabeling of ground truth data in the training datasets. Where systematic differences were lower, e.g., the ankle, differences of 1-15 mm were observed depending on the activity. Markerless motion capture represents a highly promising emerging technology that could free movement scientists from laboratory environments but 3D joint centre locations are not yet consistently comparable to marker-based motion capture.

The measurement and study of human movement is a fundamental part of many science and medicine disciplines but accurately measuring human motion is an extremely challenging task, even in highly controlled laboratory environments. Examples include, motor rehabilitation scientists and clinicians who quantify movement to inform rehabilitation design ${ }^{1,2}$ and evaluate the effects of disease and treatments ${ }^{3}$. Neuroscientists study brain-movement interaction and motor learning ${ }^{4}$. Similarly, psychologists examine human motor development ${ }^{5}$, human motor behavior $^{6}$ and the effects of psychological disorders on movement ${ }^{7}$. Sports and exercise physiologists and biomechanists examine the metabolic costs of human movement ${ }^{8}$, sports techniques ${ }^{9,10}$, injury mechanisms ${ }^{11,12}$ and equipment design ${ }^{13}$. Finally, engineers quantify movement for prosthetics ${ }^{14}$, exoskeleton ${ }^{15}$ and rehabilitation robotics design ${ }^{16}$. The scope and breadth of these examples demonstrate the substantial impact that human movement research makes to science and medicine ${ }^{17}$.

The movement sciences described above traditionally rely on vision-based tools using either regular image or video data and manually annotating points of interest or marker-based motion capture systems. Manual annotation of video data is low in cost, easy to deploy in both laboratory and real-world settings and is relatively unobtrusive $^{10}$. However, manual annotation is highly time-consuming and is liable to subjective error ${ }^{10}$, which ultimately limits how much data can be processed and the quality of subsequent analyses. Automated markerbased motion capture systems are now commonplace in laboratory environments providing marker tracking with sub-millimeter accuracy ${ }^{18}$ and greatly reducing the processing time when compared to manual data annotation. However, use of marker-based systems is limited to capturing repetitive, non-representative tasks performed in small, highly controlled environments such as laboratories ${ }^{17}$. The requirement to wear markers may also alter natural movement patterns ${ }^{10}$ and the placement of markers is subject to inter-session and inter-tester variability ${ }^{19}$. Furthermore, marker placements often do not correspond directly to the true anatomical joint centres they are representing ${ }^{20}$, and soft tissue artefact can add further measurement error ${ }^{21,22}$. These limitations of marker-based motion capture systems have been well studied in relation to "gold standard" methods such as bi-planar videoradiography, where marker-based errors up to $30 \mathrm{~mm}$ have been reported for lower limb joints centre locations ${ }^{21,22}$. However, marker-based motion capture remains the current de-facto standard for quantifying human movement

Centre for the Analysis of Motion, Entertainment Research and Applications, University of Bath, Bath, UK. ${ }^{\boxplus}$ email: In424@bath.ac.uk 
within laboratories as it is more accessible in terms of cost, flexible in terms of capture volume size and safer, than $\mathrm{x}$-ray based criterion methods.

Pose estimation is a general computer vision problem where the aim is to detect the position and orientation of an object without the placement of markers. Specifically, this process involves detecting a sparse set of keypoints that describe the object's pose. In human pose estimation, joint centres such as the hip, knee or ankle, are estimated in order to reason the position and orientation of the body. This process represents a challenging problem as the algorithm should be invariant to changes in scale, perspective, lighting and even partial occlusion of a body part. The development of large, high-quality data sets (e.g., COCO Keypoint Detection Challenge, MPII Human Pose Dataset and VGG Pose Dataset) has allowed pose estimation research to develop rapidly using supervised deep-learning methods. Typically, some implementation of a convolutional neural network (CNN) is used to learn features associated with each key-point in the training dataset. At deployment, a forward pass of an image returns a 2D confidence map of key point locations (e.g., hip or elbow joint centres) in relation to that image alone. The accuracy of these CNN based methods is typically evaluated against hand-labelled ground truth data which are undoubtably subject to human error ${ }^{10,17}$ and not necessarily a true gold standard measure.

The application of pose estimation algorithms represents an exciting development for movement scientists with the promise of freeing research designs from highly constrained laboratory-based analyses ${ }^{17}$ and allowing for data capture 'in the wild', i.e. in real world, ecologically valid environment. The prospect of using low-cost imaging systems to unobtrusively capture large amounts of data in ecologically valid settings (e.g., in clinics, homes or outdoors) opens new avenues of research with larger and more varied samples, reduced bias and ultimately datasets that better represent the phenomena being studied. But despite the potential that pose estimation presents for the study of human movement, little is known about how accurately such methods can detect human joint centres, which are a fundamental requirement in almost all human motion analysis techniques. Furthermore, to use such an approach within human movement research, considerations must also be made regarding the $3 \mathrm{D}$ reconstruction of $2 \mathrm{D}$ key-points from multiple cameras and for ensuring robust temporal continuity between frames, e.g., ensuring that key-points are associated or tracked consistently and robustly as a function of time. All of these data processing requirements are non-trivial and require substantial expertise across multiple disciplines (e.g., biomechanics, signal process, computer vision) to produce accurate and reliable markerless $3 \mathrm{D}$ representations of human movement.

Human pose estimation is a rapidly developing field of computer science research. Every year there are many releases of new pose estimation algorithms as researchers compete in challenges such as the COCO Keypoint Detection Challenge. While such challenges are designed to accelerate the advancement of pose estimation technology, it is unclear whether these advancements actually benefit pose estimation technology when applied to specific domains such as human movement sciences. It is unfeasible to evaluate every pose estimation algorithm for use in human movement sciences and as such, in this research, we focus upon three popular systems; OpenPose $^{23}$, AlphaPose ${ }^{24}$ and DeepLabCut ${ }^{25}$. OpenPose represents a bottom-up approach to multi-person pose estimation as it simultaneously detects every instance of a given body part while also associating each body part to a person via a set of $2 \mathrm{D}$ vector fields that encode the location and orientation of limbs in the image $\mathrm{e}^{23}$. The OpenPose package was selected here as it is easy to install and use as well as being one of the only systems to provide a foot detection at the time of writing. As such OpenPose is a popular system and has started to receive some attention in movement sciences research ${ }^{10,26-28}$. AlphaPose employs a top-down approach to multi-person pose estimation, first detecting individual people within an image before applying pose-estimation to detect key points of each detected person ${ }^{24}$. AlphaPose was selected as it represents a different approach to pose estimation inference (top-down) and reported improved performance over OpenPose on MPII and COCO dataset benchmarks ${ }^{24}$. Finally, DeepLabCut leverages the DeeperCut pose estimation algorithm ${ }^{29}$, data augmentation and transfer learning to allow researchers to re-train and specialise a CNN to detect user-specified key-points. DeepLabCut provides a set of tools to implement active-learning-based network refinement ${ }^{30}$ and was selected as to date, it has by far had the greatest impact upon movement sciences research ${ }^{17}$.

As with any emerging technology, validation against an established gold-standard is an important step to help researchers understand both the strengths and weaknesses of a system. Such a process permits researchers to make informed decisions about whether emerging technologies provide a suitable tool to help answer their research questions. To date, AlphaPose validation have been limited to post-training model validation, where the final model is validated using human labeled ground truth images provided within the MPII and COCO datasets. However, crowd-sourced datasets are unlikely to have been labeled with the underlying anatomical structures in mind which may reduce the quality of the ground-truth data ${ }^{31}$. DeepLabCut models are also evaluated posttraining, however, as training data are typically labelled by researchers with specific domain knowledge, this data and thus the training validation could be of a higher quality than using generic crowd-sourced datasets alone. There remains, however, a need to validate both systems against marker-based motion capture using fully-synchronised, high-speed imaging systems. Two pilot studies have attempted to evaluate the accuracy of OpenPose against marker-based motion-capture using a very small sample size $(n=2)$. Zago et al. ${ }^{27}$, evaluated 3D joint centre locations derived from a stereo-vision system and OpenPose for walking activities. Joint location differences of between 20 and $60 \mathrm{~mm}$ were reported. However, unequal sampling frequencies $(30 \mathrm{~Hz}$ for image data, $100 \mathrm{~Hz}$ for marker-based data) and an absence of synchronisation hardware may have contributed to an unknown portion of the reported differences. Nakano et al. ${ }^{28}$, tested a multi-camera based OpenPose system against marker-based motion capture and reported that $80 \%$ of joint centre differences were less than $30 \mathrm{~mm}$. Larger differences were attributed to key-point detection failures at the 2D pose detection stage. Again, there was a lack of synchronisation hardware, low and uneven sampling rates and no information detailing how the two systems Euclidean spaces were aligned making it difficult to know what proportion of the reported differences can be attributed to OpenPose. 


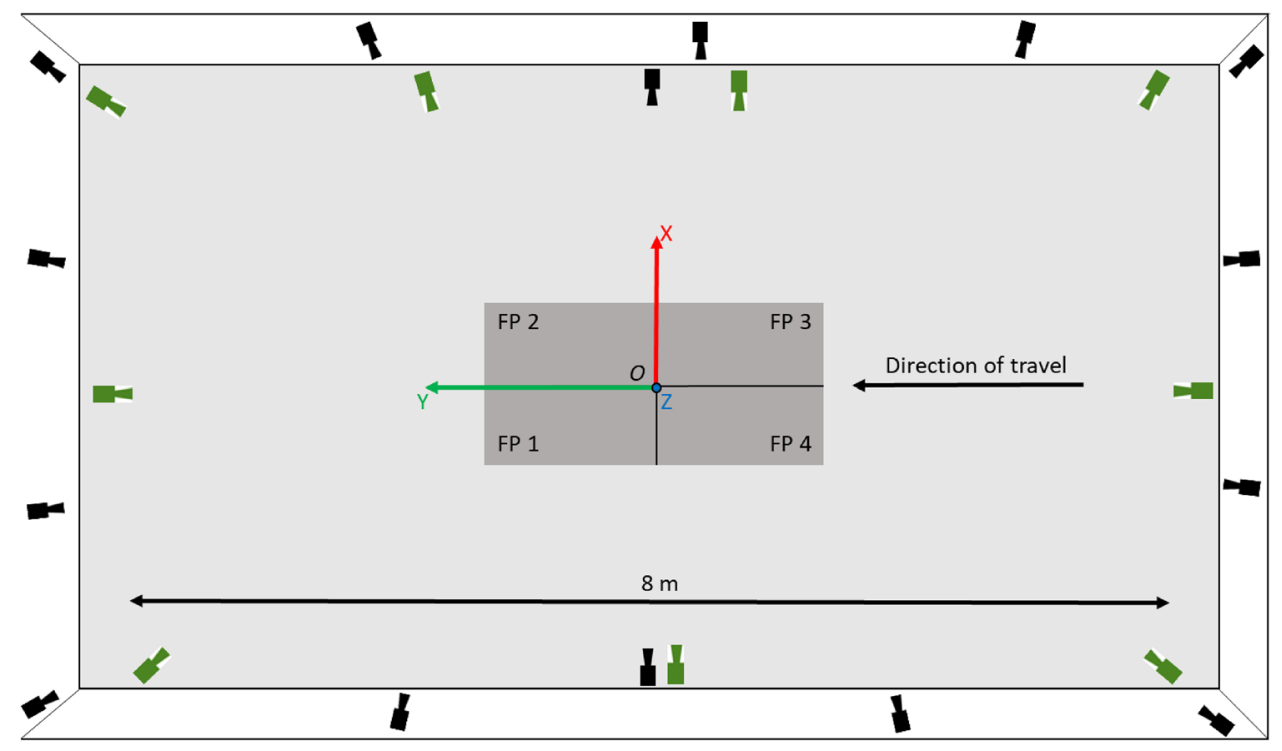

Figure 1. Birds-eye view detailing the layout of the capture volume. Four force plates (FP) are shown in the centre in dark grey. Black cameras depict Qualysis camera locations, green cameras depict JAI machine vision camera locations. The origin of a right-handed coordinate system was set in the centre of the force plates at position $\mathrm{O}$ with the positive $\mathrm{z}$-axis set normal to the $\mathrm{X}-\mathrm{Y}$ floor plane.

There is a need, therefore, to concurrently and robustly evaluate open-source pose estimation algorithms against marker-based motion capture for a range of fundamental human movements. Evaluating using a full-body biomechanical six degrees of freedom model will allow movement scientists to better understand the strengths and weakness of such methods in their current form. Furthermore, testing multiple algorithms will allow for the consistent application of tracking, 3D-fusion and system synchronisation methods, which has so far been absent from the literature to date. The aim of this study was to assess the ability of pre-trained CNN based pose estimation algorithms, when used with our tracking and 3D fusion algorithm, to accurately reconstruct the location of joint centres during fundamental human movements. The purpose of this study was to provide human movement scientists with robust evaluation data to make more informed choices about the application of these methods within their own research or practice.

\section{Methods}

Fifteen healthy participants $(7$ males $[1.82 \pm 0.11 \mathrm{~m}, 85.7 \pm 11.1 \mathrm{~kg}], 8$ females $[1.65 \pm 0.08 \mathrm{~m}, 63.2 \pm 6.0 \mathrm{~kg}]$ ) provided written informed consent. During a single testing session each participant performed ten walking trials, ten running trials (both at self-selected pace) and ten counter-movement jumps in a randomised order while wearing a full body marker-set. Movement trials were captured concurrently using two motion capture systems. Evaluation data were captured using a 15-camera marker-based motion capture system (Oqus, Qualysis $\mathrm{AB}$, Gothenburg, Sweden) while additional image data were captured using a custom 9-camera computer vision system (JAI sp5000c, JAI ltd, Denmark) (Fig. 1). Motion capture systems were time-synchronised by means of a periodic TTL-pulse generated by the custom system's master frame grabber to achieve a frame locked sampling frequency of $200 \mathrm{~Hz}$ in both systems. Additionally, a stopping trigger signal for both systems was generated by the master frame grabber. This ensured that not only did both camera systems stop recording at the same time but that frames were captured by all cameras in unison. To further ensure that synchronisations did not drift, two visible LEDs and one infra-red LED were placed in the capture volume in view of both system's cameras. The LEDs were programmed to flash in sequence and could later be used to ensure that frame alignment between systems was as expected.

The Qualysis system was calibrated as per the manufacturer's specifications. The custom camera system used observations of a binary dot matrix to initialise each camera's intrinsic parameters ${ }^{32}$, then extrinsic parameters were initialised from pairs of cameras with shared dot matrix observations. A global optimisation was performed using Sparse Bundle Adjustment ${ }^{33}$ to determine the final intrinsic and extrinsic parameters. A right-handed coordinate system was defined for both systems by placing a Qualysis L-Frame in the centre of the capture volume. To refine the alignment of each system's Euclidean space, a single marker was moved randomly through the capture volume and tracked by both systems. This marker data provided points with which the spatial alignment could be optimised in a least-squares sense. To assess the reconstruction accuracy of both systems a wand was moved through the capture volume and tracked by both systems before the mean $( \pm S D)$ resultant vector magnitude was computed and compared to the known dimensions of the wand.

To capture criterion data, a full body marker set comprising of 44 individual markers and eight clusters were attached to each participant to create a full body six degrees of freedom $(6 \mathrm{DoF})$ model (bilateral feet, shanks and thighs, pelvis and thorax, upper and lower arms, and hands) (Supplementary Materials-Fig. A1). Following labelling and gap filling of trajectories (Qualysis Track Manager v2019.3, Qualysis, Gothenburg, Sweden) data 
were exported to Visual 3D (v6, C-Motion Inc, Germantown, USA) where raw trajectories were low-pass filtered (Butterworth 4th order, cut-off $12 \mathrm{~Hz}$ ) and a $6 \mathrm{DoF}$ model was computed. The marker-based model's joint centres were computed as the point $50 \%$ between the medial and lateral marker for all joints except the hip joint centre which was computed using the regression equations reported by Bell et $\mathrm{al}^{34}$.

Multi-camera image data were processed using OpenPose (v1.6.0 $0^{23}$ ), AlphaPose (v0.3.0.24) and DeepLabCut's pre-trained human pose model $\left(\mathrm{v} 2 \cdot 1 \cdot 7^{25}\right)$. OpenPose returned a 25-point body model, AlphaPose returned an 18-point body model and DeepLabCut returned a 15-point body model (Supplementary Materials-Fig. A2 and Table A1). Temporal frame alignment between marker and markerless systems were confirmed and refined using the flashing LEDs, where required. Where multiple people were detected in the capture volume tracking or frame-to-frame association was performed using an occupancy map-based approach. Reconstruction of each joint centre in the 3D space was achieved as follows. For a given keypoint, each detection was back projected to produce a ray in the $3 \mathrm{D}$ space using the camera calibration information and accounting for non-linearities such as lens distortion. The intersection of the $3 \mathrm{D}$ rays provides the joint centre location. However, to account for $2 \mathrm{D}$ joint centre detection errors a RANSAC process determined a set of inlier and outlier rays. The final 3D joint centre solution was taken as the intersection of the remaining inlier rays. For a more detailed description of this process $\mathrm{se}^{35}$. The $3 \mathrm{D}$ joint centre reconstructions were filtered using a bi-directional Kalman filter ${ }^{36}$ before being written to C3D file format. Example videos are provided in the supplementary materials.

For walking and running trials, touch-down (TD) and toe-off (TO) events were computed using Visual 3D's 'automatic_gait_events' function ${ }^{37}$ which was applied to marker-based data before each step cycle was registered to 101 points from TD to the next corresponding TD. As marker and markerless data were temporally synchronised, events derived from marker-based data could be used for markerless data too, thus ensuring event timing consistency between methods. For each trial, an average of four and six complete step cycles were captured for walking and running, respectively. Jumping trials were registered to 101 points from first movement to stabilisation. Where first movement was defined as the point that the vertical force dropped below body weight for 20 consecutive frames and stabilisation was defined as the point that vertical force remained within 3 standard deviations of bodyweight.

Differences in joint centre trajectories between the marker-based and markerless joint centres were determined by computing the 3D Euclidean distance at each time point. Additionally, the signed differences between trajectories were computed along each global coordinate system axis (X-axis: anterior-posterior, Y-axis: medial-lateral, Z-axis: superior-inferior). Agreement between methods was evaluated using Bland-Altman analysis and linear regression models. Normality was tested using a Shapiro-Wilk test. Bland-Altman analysis permits the delineation of systematic (bias) and random (standard deviation of bias) difference between measures with $95 \%$ limits of agreement $(\mathrm{LoA})^{38}$. Where data were not found to be normally distributed, non-parametric LoA were computed using the 5 th and 95 th percentiles ${ }^{39}$. Additionally, we computed linear regression models which provide reliable and sensitive means to compare between biomechanical waveforms ${ }^{40}$. The coefficient of determination $\left(\mathrm{R}^{2}\right)$ indicates the strength of the linear relationship between the two measures while the intercept indicates the shift or offset and the gradient describes the variation of one waveform relative to another ${ }^{40}$.

Ethical approval. The study was conducted according to the guidelines of the Declaration of Helsinki, and approved by the Institutional Review Board (or Ethics Committee) of the University of Bath (EP1819052 25/07/19).

\section{Results}

Example joint centre trajectories derived from each motion capture method for a single participant during walking, running and jumping are provided in Fig. 2. Further examples for other joint centres are provided in the supplementary materials (Figs. A3-A5). For each pose estimation method, mean \pm SD time-series differences for the hip joint centre, when compared to marker-based motion capture, are shown in Fig. 3. Further joint centre differences are provided in the supplementary materials (Figs. A6-A8).

For all pose estimation methods and all activities, the lowest systematic differences were observed at the ankle joint centre (Table 1) with random error and LoA also smallest at this joint. The hip and knee joint centres displayed the largest systematic differences for all pose estimation methods (Table 1 and Fig. 4) with knee demonstrating higher random error and LoA during walking and running. The knee also presented higher random error and LoA during jumping. Shoulder joint centre mean differences were typically observed to be larger than those at the ankle but lower than the hip or knee with random error and LoA following the same trend. OpenPose difference distributions for each joint centre are provided in Fig. 4 with further examples for AlphaPose and DeepLabCut provided in the supplementary materials (Figs. A11, A12).

Differences in pose estimation performance were observed between walking, running and jumping (Fig. 5). For walking and running, the smallest systematic and random differences were observed for OpenPose and largest for DeepLabCut. However, during jumping OpenPose and AlphaPose mean differences and random errors were comparable between markerless methods (within $\sim 1 \mathrm{~mm}$ of each other) (Fig. A10. While the largest systematic differences, random errors and LoA were generally observed for DeepLabCut, the results for ankle and shoulder during jumping were comparable to those observed for OpenPose and AlphaPose. Further visual examples are provided as videos in the supplementary materials ${ }^{41}$.

\section{Discussion}

This is the first study to robustly assess the ability of multiple pre-trained CNN based pose estimation algorithms (OpenPose ${ }^{23}$, AlphaPose ${ }^{24}$ and DeepLabCut's ${ }^{25}$ pre-trained human pose model) to accurately reconstruct the location of joint centres against high quality ground truth data (biomechanics marker-based model). Markerless 

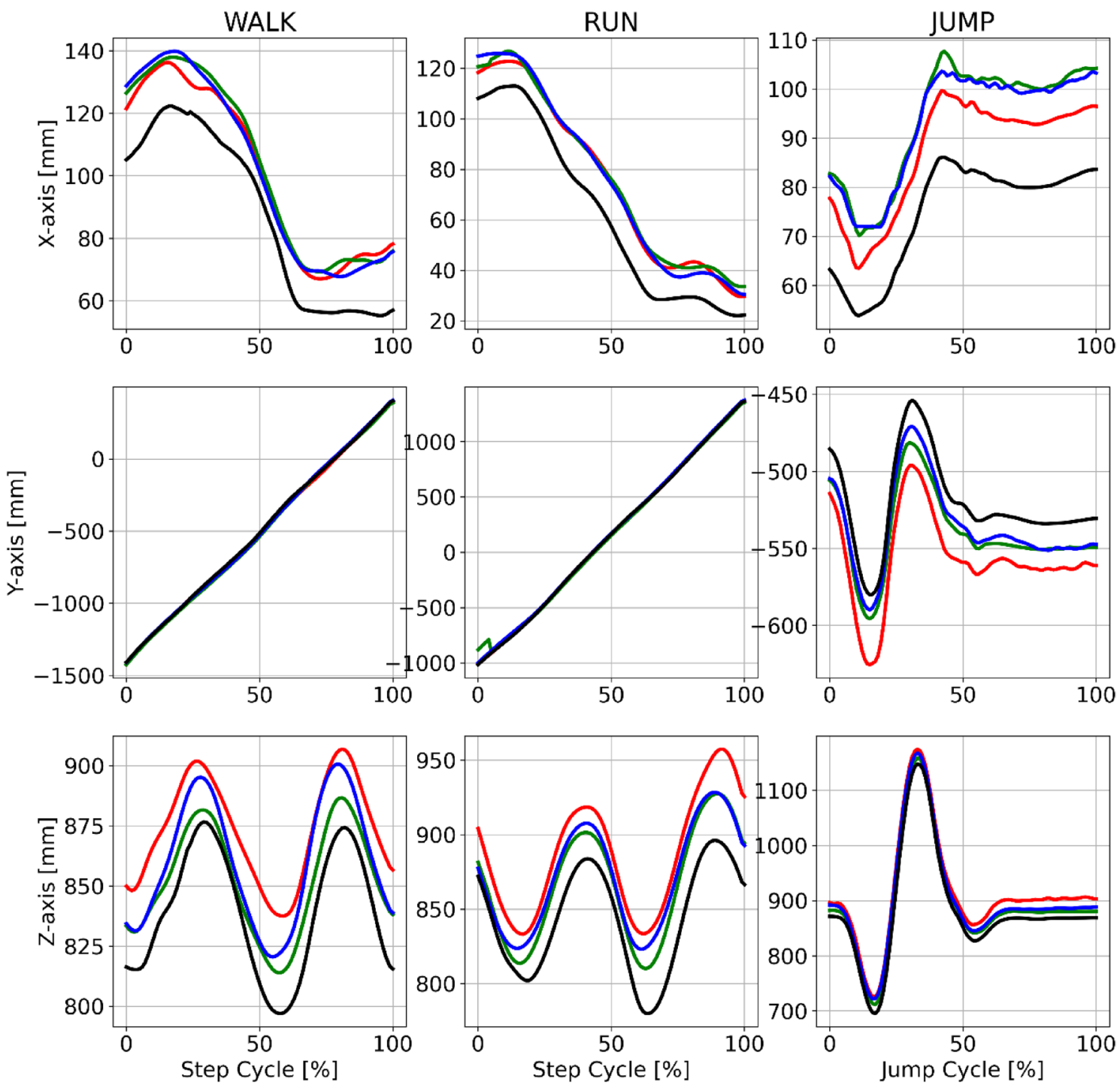

Figure 2. Example mean right hip joint centre trajectories for a single participant (P10) during walking (left), running (centre) and jumping (right) in the global coordinate system axes. Marker based trajectories (black), OpenPose (green), AlphaPose (blue) and DeepLabCut (red). X-axis = medial-lateral. Y-axis = anterior-posterior. $\mathrm{Z}$-axis $=$ superior-inferior.

pose estimation algorithms show great potential to facilitate large scale movement studies in a range of environments and the results of this study align with others ${ }^{28}$ in demonstrating that the resulting $3 \mathrm{D}$ skeletons can under favorable conditions, produce visually impressive outcomes using several different pose estimation methods. However, for all three pose estimation methods during all activities (walking, running and jumping), there was clear evidence of both systematic and random differences when compared to marker-based motion capture (Table 1, A2-A4), which suggests that such technology may require further development before its performance surpasses the current de-facto methods (marker-based motion capture) in human movement sciences.

The largest differences between pose estimation derived 3D joint centres were observed at the hip, where mean differences ranged between $29 \mathrm{~mm}$ (OpenPose during running) and $53 \mathrm{~mm}$ (DeepLabCut during running). Bland-Altman analysis revealed that these differences were largely systematic in nature with all pose estimation methods placing the hip joint centres in a more lateral and inferior position than those derived from markers placed on the pelvis (Tables A2, A4 and A6). Additionally, systematic differences were observed at the knee joint, most notably in the vertical axis where all pose estimation methods placed the joint centre between $\sim 20$ and $30 \mathrm{~mm}$ below the marker derived joint centre. Deep learning-based pose estimation methods are typically trained using supervised learning techniques where annotated images provide training examples of the desired joint centre locations. Systematic differences in joint centre locations such as those observed here are likely a result of large-scale mislabeling of hip joint centre locations in the datasets used to train each deep learning model. At best, we can expect that supervised deep learning models will be able to perform as well as but not better than the data with which they have been trained ${ }^{42}$. Consequently, it is unrealistic to expect a pose estimation model trained on open access data sets such as $\mathrm{COCO}^{43}$ or MPII ${ }^{44}$ to estimate the location of anatomically accurate joint centres when as this study suggests, such joint centres are not accurately labelled in the training data. There 

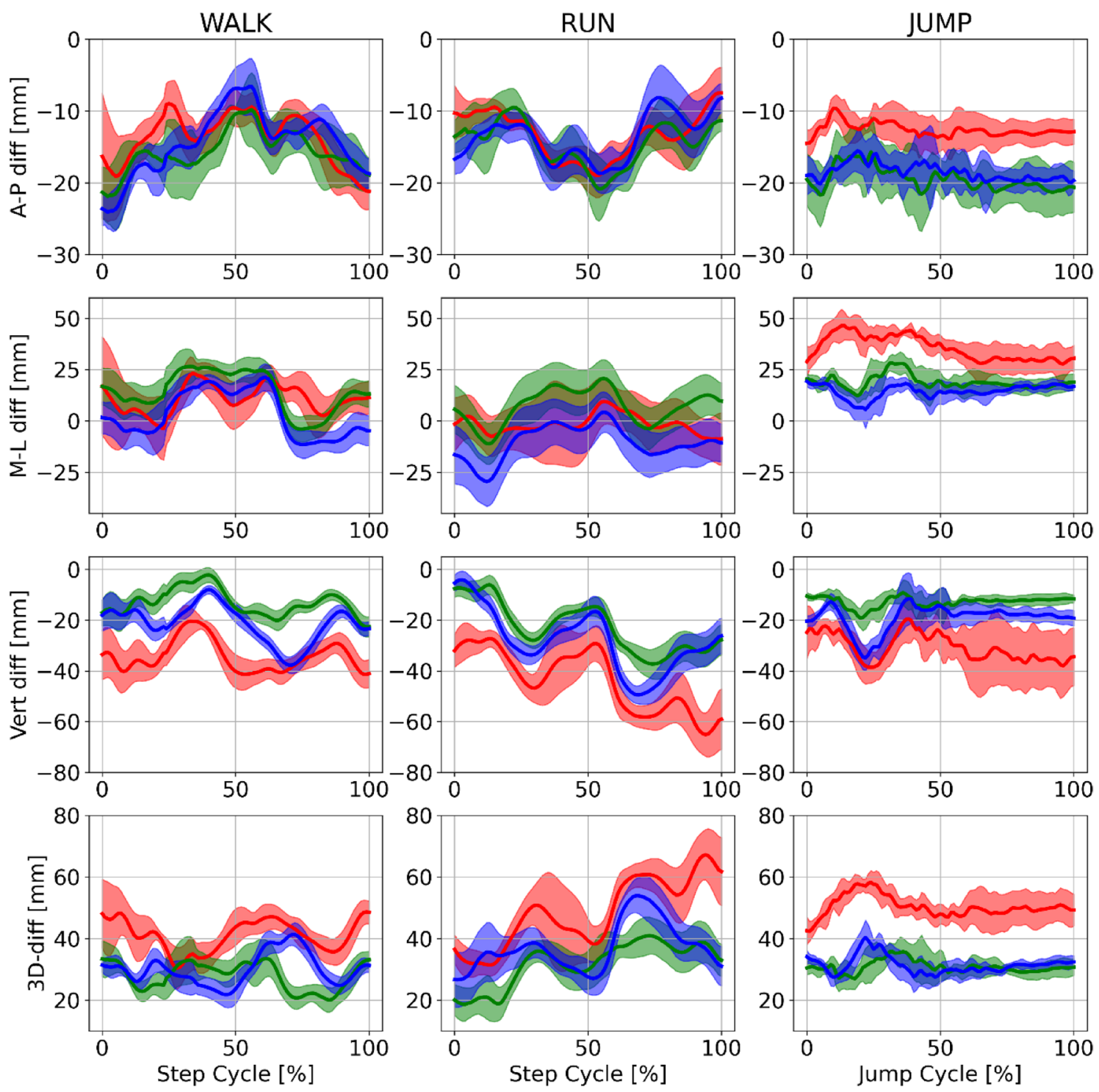

Figure 3. Example mean $( \pm S D)$ differences between marker based and markerless trajectories for the right hip joint centre trajectories of a single participant (P10) during walking (left), running (centre) and jumping (right). Marker based trajectories (black), OpenPose (green), AlphaPose (blue) and DeepLabCut (red). Row $1=$ anterior-posterior differences. Row $2=$ medial-lateral. Row $3=$ superior-inferior differences. Row $4=3 \mathrm{D}$ Euclidean differences.

is a need, therefore, for the development of large scale, open access pose estimation data sets that have been labelled by individuals with detailed knowledge of human anatomy. Although systematic and random errors were largest for DeepLabCut, the software provides functionality for leveraging transfer learning, which helps specialise pre-trained networks using small amounts of additional training data. As such, we would expect to see improved results for DeepLabCut if additional data and training time are leveraged on top of the DeepLabCut pre-trained human pose model that was evaluated in this study. The same could also be achieved with AlphaPose and OpenPose, however more in-depth deep learning expertise would be required to achieve this and the present study was concerned with the performance of pre-trained models in their 'off the shelf' form. Indeed, previous work utilising re-trained DeepLabCut has demonstrated promising 2D sagittal plane results during underwater running with mean differences of approximately $10 \mathrm{~mm}^{45}$.

The smallest differences were observed for the ankle joint, which demonstrated considerably lower systematic differences and random errors when compared to the hip and knee joint centres. The results observed for the ankle joint centre, which is perhaps easier to identify and label than the hip and knee demonstrate the potential that, given (conceivably more) anatomically accurate training data, pose estimation methods could achieve for all joint centres. Indeed, mean differences as low as $2 \pm 7 \mathrm{~mm}$ (Tables A4 and A5) were observed using OpenPose during jumping and represent values that are well within the known error ranges $(25-33 \mathrm{~mm})$ of marker-based motion capture techniques ${ }^{21,22}$. Once all joints can be estimated to this level of accuracy the focus of attention instead turns to between session repeatability ${ }^{46}$, which can be problematic for marker-based systems when markers are not placed in identical locations between sessions ${ }^{47}$. 


\begin{tabular}{|c|c|c|c|c|c|c|c|c|c|c|c|c|c|}
\hline & & \multicolumn{3}{|c|}{$\begin{array}{l}\text { Mean difference } \\
(\text { Bias })(\mathrm{mm})\end{array}$} & \multicolumn{3}{|c|}{$\begin{array}{l}\text { Random error } \\
( \pm \text { SD of Bias })\end{array}$} & \multicolumn{6}{|c|}{$\begin{array}{l}\text { 95\% LoA (Lower-Upper limi } \\
{[\mathrm{mm}] \text { ) }}\end{array}$} \\
\hline & & \multirow{2}{*}{\begin{tabular}{|l|} 
OP \\
29 \\
\end{tabular}} & \multirow{2}{*}{\begin{tabular}{|l|} 
AP \\
30 \\
\end{tabular}} & \multirow{2}{*}{\begin{tabular}{|l|} 
DLC \\
24
\end{tabular}} & \multirow{2}{*}{\begin{tabular}{|l|} 
OP \\
8 \\
\end{tabular}} & \multirow{2}{*}{\begin{tabular}{|l|} 
AP \\
7 \\
\end{tabular}} & \multirow{2}{*}{\begin{tabular}{|l|} 
DLC \\
8
\end{tabular}} & \multicolumn{2}{|c|}{ OP } & \multicolumn{2}{|l|}{ AP } & \multicolumn{2}{|c|}{ DLC } \\
\hline \multirow{4}{*}{ WALK } & Shoulder & & & & & & & 18 & 43 & 21 & 42 & 14 & 37 \\
\hline & Hip & 34 & 31 & 43 & 12 & 9 & 14 & 14 & 53 & 15 & 45 & 21 & 68 \\
\hline & Knee & 30 & 31 & 42 & 11 & 15 & 26 & 15 & 51 & 13 & 61 & 17 & 84 \\
\hline & Ankle & 16 & 19 & 30 & 8 & 14 & 39 & 6 & 32 & 6 & 50 & 8 & 65 \\
\hline \multirow{4}{*}{ RUN } & Shoulder & 27 & 31 & 29 & 9 & 11 & 13 & 13 & 43 & 15 & 50 & 10 & 51 \\
\hline & Hip & 29 & 32 & 45 & 9 & 10 & 21 & 14 & 45 & 16 & 50 & 18 & 78 \\
\hline & Knee & 41 & 48 & 58 & 17 & 26 & 36 & 19 & 74 & 17 & 97 & 22 & 116 \\
\hline & Ankle & 23 & 36 & 52 & 14 & 24 & 68 & 6 & 50 & 9 & 79 & 11 & 121 \\
\hline \multirow{4}{*}{ JUMP } & Shoulder & 32 & 34 & 33 & 11 & 12 & 11 & 16 & 53 & 18 & 56 & 19 & 53 \\
\hline & Hip & 36 & 36 & 53 & 11 & 10 & 15 & 18 & 54 & 20 & 51 & 29 & 78 \\
\hline & Knee & 29 & 27 & 35 & 8 & 9 & 9 & 15 & 44 & 14 & 43 & 20 & 49 \\
\hline & Ankle & 14 & 14 & 15 & 6 & 7 & 8 & 6 & 25 & 4 & 27 & 6 & 29 \\
\hline
\end{tabular}

Table 1. Bland-Altman analysis results of 3D Euclidean differences during walking, running and jumping. $\mathrm{OP}=$ OpenPose, $\mathrm{AP}=$ AlphaPose, $\mathrm{DLC}=$ DeepLabCut .
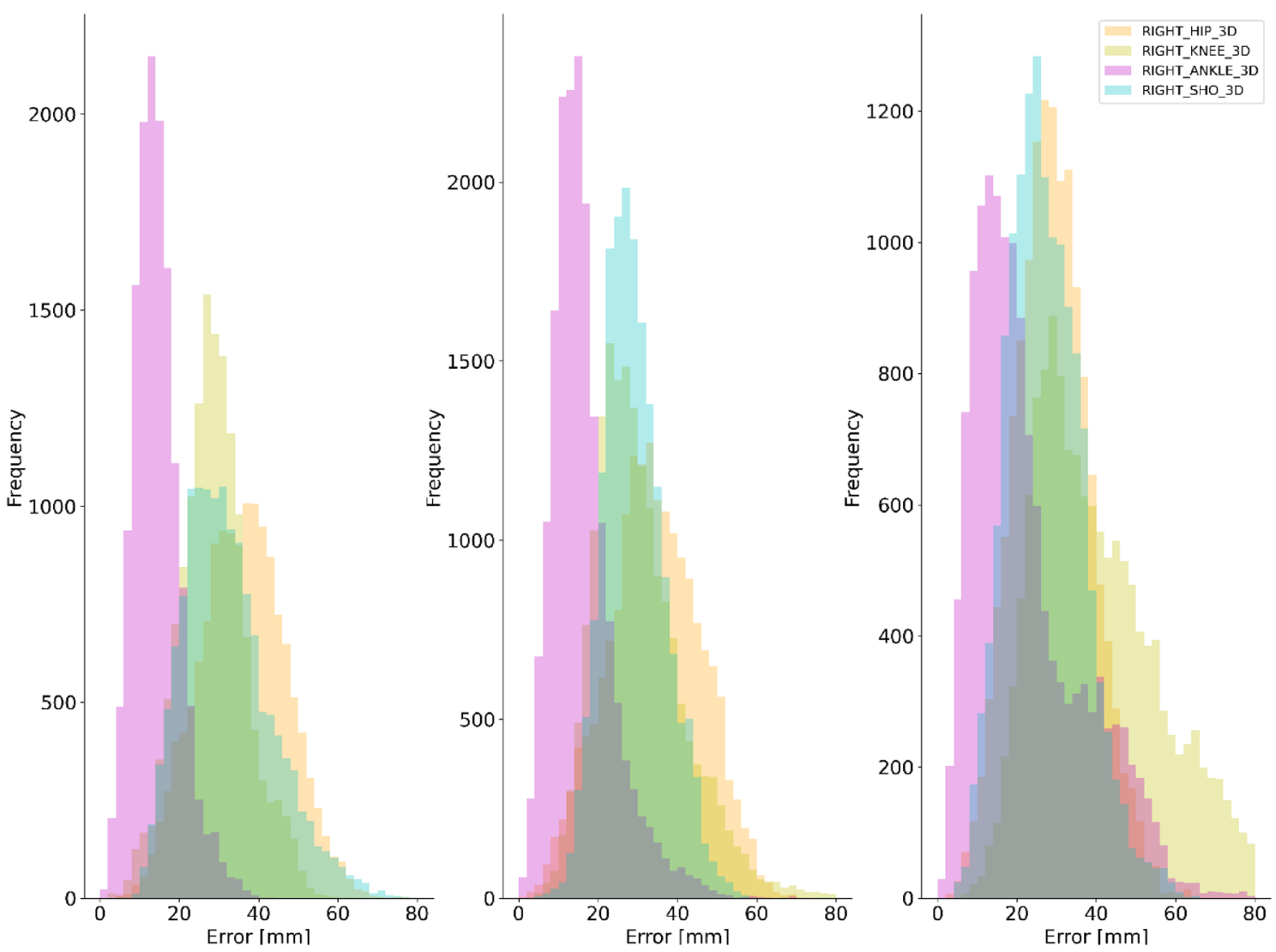

Figure 4. Example difference distributions for OpenPose at the right shoulder, hip, knee and ankle joint centres for jumping (left), walking (centre) and running (right).

Evidence of activity specific pose estimation performance was observed, with the largest mean differences and random error measured during running and the smallest mean differences and random error measured during jumping. Lower pose estimation performance during running may be attributed to the greater range of observed limb configurations and segment velocities when compared to jumping and aligns with previous evidence that pose estimation performance is highly task specific ${ }^{35}$-an important consideration for researchers. Furthermore, greater limb velocities may introduce image noise in the form of motion blur, making it harder for pose estimation methods to detect image features that represent a given joint centre. Indeed, it is unlikely that current datasets include training examples with any degree of motion blur, a factor which should be considered 

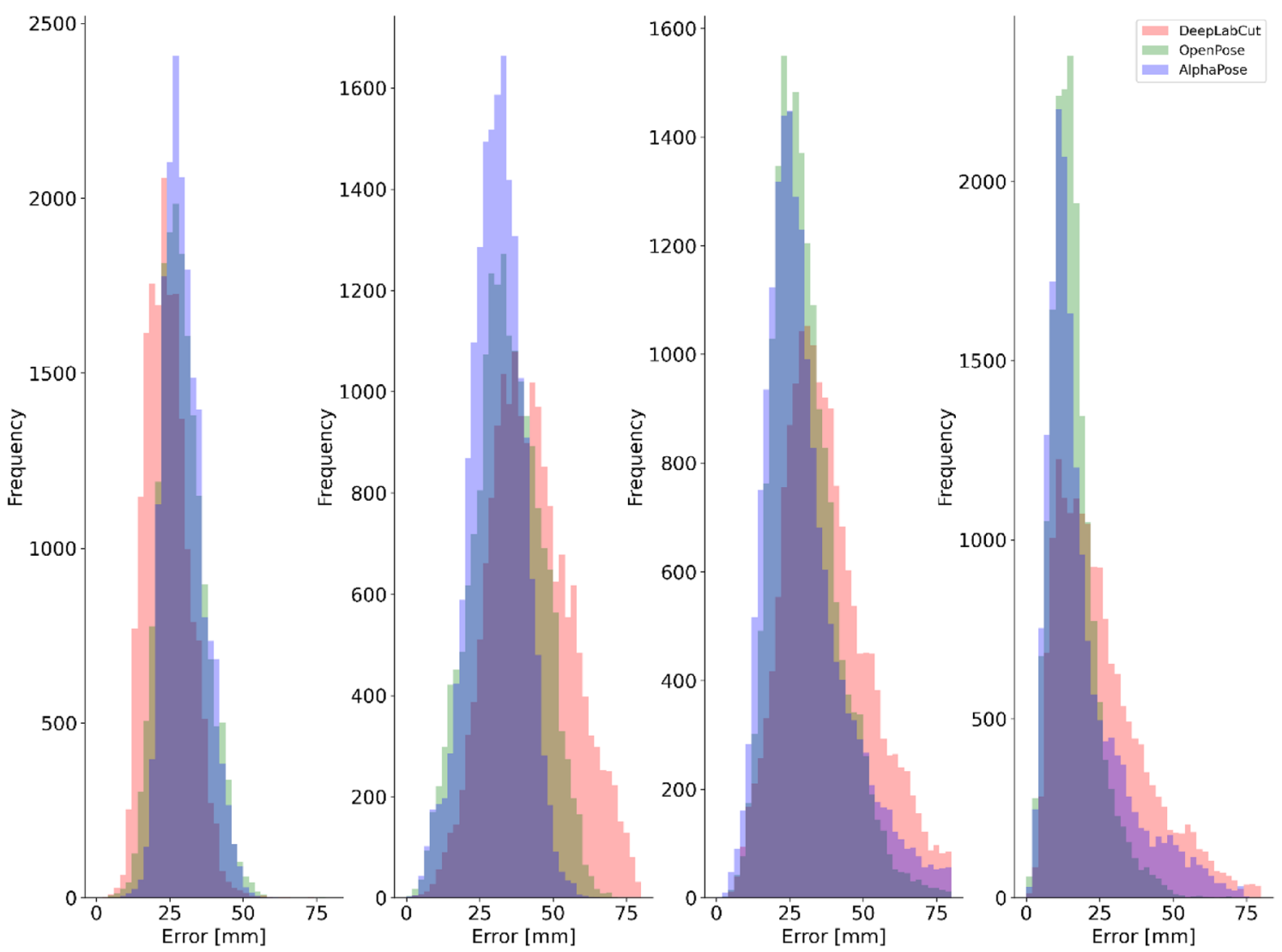

Figure 5. Difference distributions during walking for each pose estimation method at the right shoulder (left), hip (centre-left), knee (centre-right) and ankle (right) joint centres.

during the development of future datasets. In this study, we minimised noise from motion blur by utilising high frame rates $(200 \mathrm{~Hz})$, low exposure times and high-quality studio lighting. Yet, there exists a trade-off between capturing the highest quality images and using pose estimation models that can handle less than perfect images, which researchers should carefully consider as part of their research design.

Performance of OpenPose and AlphaPose across activities and joints was comparable to one another for systematic differences (between method differences $\sim 1-5 \mathrm{~mm}$ ) and random errors (between method differences $\sim 1-3 \mathrm{~mm}$ ) while larger systematic and random errors were observed for DeepLabCut. Despite using distinctly different network architectures and approaches to pose estimation, the comparable performances of OpenPose and AlphaPose were surprising. AlphaPose has previously demonstrated superior performance on computer vision benchmarks such as COCO and MPII. However, as the results of this study demonstrate, pose estimation performance should not be solely based upon these computer vision benchmarks but additionally, domain specific benchmarks using appropriate ground truth information are required ${ }^{17}$ to enhance their development and ability to generalise effectively in real-world applications. DeepLabCut did not perform as well as OpenPose and AlphaPose. DeepLabCut uses DeeperCut ${ }^{29}$ to perform pose estimation, which is the oldest method and lowest scoring (on MPII benchmark) method tested in this study and may partially explain the lower performance. An additional source of error that was unique to DeepLabCut related to single and multi-person detection capabilities. The pre-trained human pose model used in this study only returned pose information for a single person. When multiple people were in the field of view, there was a tendency for joint centres to jump between the study participant in the foreground and people in the background (supplementary video-DLC_2Dv3D). Such outliers were largely removed during 3D fusion, however it is inevitable that this issue will have contributed at times to the overall differences that were observed for DeepLabCut. It is worth noting, however, that DeepLabCut provides comprehensive tools for labelling additional data and re-training their pose estimation models. As mentioned previously, by leveraging DeepLabCut's transfer learning capabilities we would expect to see substantially improved results on those presented in this study. However, the aim of this study was to assess the current capabilities of pre-trained pose estimation models. For all pose estimation methods, our results include larger errors caused by issues such as false positive detections, tracking failures and erroneous switching of limbs. While previous research has suggested manually correcting errors ${ }^{28}$, we did not feel that this approach provided the full picture regarding pose estimation. Rather it is important for those applying pose estimation methods to be fully aware of the issues discussed in this study that require effective detection and correction during the data processing pipeline.

Aligning with previous studies examining OpenPose vs marker-based motion capture ${ }^{27,28}$, we have shown promising face validity for $3 \mathrm{D}$ joint centre locations detected using OpenPose, AlphaPose and DeepLabCut 
but results were not consistently comparable to marker-based motion capture. It is not possible to state that the results of this study meet the accuracy requirements for all human movement science studies, as such requirements will vary greatly between applications and researchers should consider carefully how appropriate the use of current markerless methods are within the context of their research questions. However, our results for joint centres such as the ankle and shoulder do fall within the known range of error for marker-based motion capture $^{21}$ demonstrating that markerless technology has a promising future in human movement science research. It is important to acknowledge that while we assume that the majority of differences reported arise from the $2 \mathrm{D}$ pose estimation process, an unknown proportion of that error could be attributed to the tracking and 3D fusion process. However, when compared to a simple triangulation process (e.g. ${ }^{28}$ ) our approach demonstrates substantially greater joint centre accuracy and robustness to outlier $2 \mathrm{D}$ joint centre detections (supplementary video-DLC_RECONSTRUCTION_ROBUST_VS_NON.mp4).

There are three key areas to consider for future development of markerless motion capture. Firstly, the need for domain specific datasets which contain anatomically accurate labels and contain representative images for the activity of interest. Additionally, ensuring that each segment has at least three non-colinear keypoints will better facilitate $6 \mathrm{DoF}$ pose estimation. Secondly, the need for domain specific benchmarks ${ }^{17}$ that go beyond the current computer vision benchmarks to test pose estimation methods on domain specific ground truth data. For example, in this study we benchmarked against validated marker-based joint centre estimation methods rather than hand labelled joint centre annotations. Without these two considerations (datasets and benchmarks) we will not see significant progress for pose estimation in the movement sciences. Finally, using modelling methods such as inverse kinematics optimisation ${ }^{48}$, which is widely established in many parts of human movement sciences, we would expect that the $3 \mathrm{D}$ joint centres derived from pose estimation methods, such as those presented in this study, could be used to provide improved estimates of $3 \mathrm{D}$ joint centres and segment kinematics. It is important to reiterate that the evaluation measure used in this study (marker-based motion capture) does not represent the true criterion measure (bi-planar videoradiography) and findings should be considered in this context. However, it is indeed promising that our results often fall within the known error ranges of marker-based motion capture and future developments should only strengthen the capabilities of markerless motion capture to free researchers from the laboratory to perform large scale studies outside of laboratory environments.

\section{Conclusions}

In this study we demonstrated that OpenPose, AlphaPose and DeepLabCut when used with our 3D fusion methods can be used to detect and reconstruct markerless 3D joint centre locations. When compared to markerbased motion capture, systematic differences were observed at the hip and knee ( 30-50 mm for all methods), most likely due to mislabeling of ground truth data in the training datasets. Where systematic differences were lower, e.g., at the ankle, differences of $1-15 \mathrm{~mm} \pm 10 \mathrm{~mm}$ were observed. OpenPose and AlphaPose demonstrated comparable performance to one another and outperformed DeepLabCut. Markerless pose estimation using the methods described in this study do not yet match the performance of marker-based motion capture at all joint centres; however, markerless motion capture can facilitate opportunities for researchers who are willing to accept a small decrease in pose estimation performance in order to capture more ecologically valid human movement.

Received: 22 April 2021; Accepted: 7 October 2021

Published online: 19 October 2021

\section{References}

1. Mundermann, L., Corazza, S. \& Andriacchi, T. P. The evolution of methods for the capture of human movement leading to markerless motion capture for biomechanical applications. J. Neuroeng. Rehabilitat. https://doi.org/10.1186/1743-0003-3-6 (2006).

2. Bates, N. A., Myer, G. D., Shearn, J. T. \& Hewett, T. E. Anterior cruciate ligament biomechanics during robotic and mechanical simulations of physiologic and clinical motion tasks: A systematic review and meta-analysis. Clin. Biomech. 30, 1-13. https://doi. org/10.1016/j.clinbiomech.2014.12.006 (2015).

3. Fern'ndez-Baena, A., Susin, A. \& Lligadas, X. Biomechanical validation of upper-body and lower-body joint movements of kinect motion capture data for rehabilitation treatments. 2012 4th International Conference on Intelligent Networking and Collaborative Systems (INCoS 2012), 656-661. https://doi.org/10.1109/iNCoS.2012.66 (2012).

4. Churchland, M. M. et al. Neural population dynamics during reaching. Nature 487, 51. https://doi.org/10.1038/nature11129 (2012).

5. Newell, K. M. MOTOR SKILL ACQUISITION. Rosenzweig, M. R. and L. W. Porter (Ed.). Annual Review of Psychology, Vol. 42. Ix+600p. Annual Reviews Inc.: Palo Alto, California, USA. Illus, 213-237 (1991).

6. Newell, K. M., Mayer-Kress, G. \& Liu, Y.-T. Aging, time scales, and sensorimotor variability. Psychol. Aging 24, 809-818. https:// doi.org/10.1037/a0017911 (2009).

7. Vaillancourt, D. E. \& Newell, K. M. Changing complexity in human behavior and physiology through aging and disease. Neurobiol. Aging 23, 1-11. https://doi.org/10.1016/s0197-4580(01)00247-0 (2002).

8. Bhargava, L. J., Pandy, M. G. \& Anderson, F. C. A phenomenological model for estimating metabolic energy consumption in muscle contraction. J. Biomech. 37, 81-88. https://doi.org/10.1016/s0021-9290(03)00239-2 (2004).

9. Hay, J. The Biomechanics of Sports Techniques. (Prentice-Hall, 1978).

10. Colyer, S. L., Evans, M., Cosker, D. P. \& Salo, A. I. T. A review of the evolution of vision-based motion analysis and the integration of advanced computer vision methods towards developing a markerless system. Sports Med. Open https://doi.org/10.1186/ s40798-018-0139-y (2018).

11. Bahr, R. \& Krosshaug, T. Understanding injury mechanisms: A key component of preventing injuries in sport. Br. J. Sports Med. 39, 324-329. https://doi.org/10.1136/bjsm.2005.018341 (2005).

12. Pavei, G. \& Minetti, A. E. Hopping locomotion at different gravity: Metabolism and mechanics in humans. J. Appl. Physiol. 120, 1223-1229. https://doi.org/10.1152/japplphysiol.00839.2015 (2016).

13. Stefanyshyn, D. J. \& Wannop, J. W. Biomechanics research and sport equipment development. Sports Eng. 18, 191-202. https:// doi.org/10.1007/s12283-015-0183-5 (2015). 
14. Zelik, K. E. et al. Systematic variation of prosthetic foot spring affects center-of-mass mechanics and metabolic cost during walking. IEEE Trans. Neural Syst. Rehabil. Eng. 19, 411-419. https://doi.org/10.1109/tnsre.2011.2159018 (2011).

15. Zoss, A. B., Kazerooni, H. \& Chu, A. Biomechanical design of the Berkeley lower extremity exoskeleton (BLEEX). IEEE Asme Trans. Mechatron. 11, 128-138. https://doi.org/10.1109/tmech.2006.871087 (2006).

16. Freeman, C., Exell, T., Meadmore, K., Hallewell, E. \& Hughes, A.-M. Computational models of upper-limb motion during functional reaching tasks for application in FES-based stroke rehabilitation. Biomed. Eng. Biomedizinische Technik 60, 179-191. https:// doi.org/10.1515/bmt-2014-0011 (2015).

17. Seethapathi, N., Shaofei, W., Saluja, R., Blohm, G. \& Kording, K. P. Movement science needs different pose tracking algorithms arXiv. arXiv, 13 pp.-13 pp. (2019).

18. Topley, M. \& Richards, J. G. A comparison of currently available optoelectronic motion capture systems. J. Biomech. https://doi. org/10.1016/j.jbiomech.2020.109820 (2020).

19. Tsushima, H., Morris, M. E. \& McGinley, J. Test-retest reliability and inter-tester reliability of kinematic data from a threedimensional gait analysis system. J. Jpn. Phys. Therapy Assoc. Rigaku ryoho 6, 9-17. https://doi.org/10.1298/jipta.6.9 (2003).

20. Cappozzo, A., Catani, F., Della Croce, U. \& Leardini, A. Position and orientation in-space of bones during movement-anatomical frame definition and determination. Clin. Biomech. 10, 171-178. https://doi.org/10.1016/0268-0033(95)91394-t (1995).

21. Miranda, D. L., Rainbow, M. J., Crisco, J. J. \& Fleming, B. C. Kinematic differences between optical motion capture and biplanar videoradiography during a jump-cut maneuver. J. Biomech. 46, 567-573 (2013).

22. Kessler, S. E. et al. A direct comparison of biplanar videoradiography and optical motion capture for foot and ankle kinematics. Front. Bioeng. Biotechnol. 7, 1. https://doi.org/10.3389/fbioe.2019.00199 (2019).

23. Cao, Z., Simon, T., Wei, S.-E., Sheikh, Y. \& Ieee. in 30th IEEE/CVF Conference on Computer Vision and Pattern Recognition (CVPR). $1302-1310$ (2017).

24. Fang, H.-S., Xie, S., Tai, Y.-W., Lu, C. \& Ieee. in 16th IEEE International Conference on Computer Vision (ICCV). 2353-2362 (2017).

25. Mathis, A. et al. DeepLabCut: Markerless pose estimation of user-defined body parts with deep learning. Nat. Neurosci. $21,1281$. https://doi.org/10.1038/s41593-018-0209-y (2018).

26. Slembrouck, M. et al. Multiview 3D Markerless Human Pose Estimation from OpenPose Skeletons. Advanced Concepts for Intelligent Vision Systems. 20th International Conference, ACIVS 2020. Proceedings. Lecture Notes in Computer Science (LNCS 12002), 166-178. https://doi.org/10.1007/978-3-030-40605-9_15 (2020).

27. Zago, M. et al. 3D tracking of human motion using visual skeletonization and stereoscopic vision. Front. Bioeng. Biotechnol. https:// doi.org/10.3389/fbioe.2020.00181 (2020).

28. Nakano, N. et al. Evaluation of 3D markerless motion capture accuracy using openpose with multiple video cameras. Front. Sports Active Living 2, 50-50. https://doi.org/10.3389/fspor.2020.00050 (2020).

29. Insafutdinov, E., Pishchulin, L., Andres, B., Andriluka, M. \& Schiele, B. DeeperCut: A deeper, stronger, and faster multi-person pose estimation model. Comput. Vis. Eccv 9910, 34-50. https://doi.org/10.1007/978-3-319-46466-4_3 (2016).

30. Nath, T. et al. Using DeepLabCut for 3D markerless pose estimation across species and behaviors. Nat. Protoc. 14, $2152-2176$. https://doi.org/10.1038/s41596-019-0176-0 (2019).

31. Goodman, J. K., Cryder, C. E. \& Cheema, A. Data collection in a flat world: The strengths and weaknesses of mechanical Turk samples. J. Behav. Decis. Mak. 26, 213-224. https://doi.org/10.1002/bdm.1753 (2013).

32. Zhang, Z. Y. A flexible new technique for camera calibration. IEEE Trans. Pattern Anal. Mach. Intell. 22, 1330-1334. https://doi. org/10.1109/34.888718 (2000).

33. Triggs, B., Zisserman, A. \& Szeliski, R. Vision Algorithms: Theory and Practice: International Workshop on Vision Algorithms Corfu, Greece, September 21-22, 1999 Proceedings. (Springer, 2003).

34. Bell, A. L., Brand, R. A. \& Pedersen, D. R. Prediction of hip-joint center location from external landmarks. Hum. Mov. Sci. 8, 3-16. https://doi.org/10.1016/0167-9457(89)90020-1 (1989).

35. Needham, L., Evans, M., Cosker, D. P. \& Colyer, S. L. Can markerless pose estimation algorithms estimate 3D mass centre positions and velocities during linear sprinting activities?. Sensors 21, 2889 (2021).

36. Rauch, H. E., Tung, F. \& Striebel, C. T. Maximum likelihood estimates of linear dynamic systems. AIAA J. 3, 1445-1450. https:// doi.org/10.2514/3.3166 (1965).

37. Stanhope, S. J., Kepple, T. M., McGuire, D. A. \& Roman, N. L. Kinematic-based technique for event time determination during gait. Med. Biol. Eng. Compu. 28, 355-360. https://doi.org/10.1007/bf02446154 (1990).

38. Atkinson, G. \& Nevill, A. M. Statistical methods for assessing measurement error (reliability) in variables relevant to sports medicine. Sports Med. 26, 217-238. https://doi.org/10.2165/00007256-199826040-00002 (1998).

39. Bland, J. M. \& Altman, D. G. Measuring agreement in method comparison studies. Stat. Methods Med. Res. 8, 135-160. https:// doi.org/10.1191/096228099673819272 (1999).

40. Iosa, M. et al. Assessment of Waveform Similarity in Clinical Gait Data: The Linear Fit Method. Biomed Research International 2014, https://doi.org/10.1155/2014/214156 (2014).

41. Andriluka, M., Pishchulin, L., Gehler, P., \& Schiele, B.

42. Goodfellow, I., Bengio, Y. \& Courville, A. Deep Learning. Deep Learning, 1-775 (2016).

43. Lin, T.-Y. et al. Microsoft COCO: Common objects in context. Comput. Vis. Eccv 8693, 740-755. https://doi.org/10.1007/978-3319-10602-1_48 (2014).

44. Andriluka, M., Pishchulin, L., Gehler, P., Schiele, B. \& Ieee. in 27th IEEE Conference on Computer Vision and Pattern Recognition (CVPR). 3686-3693 (2014).

45. Cronin, N. J., Rantalainen, T., Ahtiainen, J. P., Hynynen, E. \& Waller, B. Markerless 2D kinematic analysis of underwater running: A deep learning approach. J. Biomech. 87, 75-82. https://doi.org/10.1016/j.jbiomech.2019.02.021 (2019).

46. Kanko, R. M., Laende, E., Selbie, W. S. \& Deluzio, K. J. Inter-session repeatability of markerless motion capture gait kinematics. J. Biomech., 110422 (2021).

47. Noehren, B., Manal, K. \& Davis, I. Improving between-day kinematic reliability using a marker placement device. J. Orthop. Res. 28, 1405-1410. https://doi.org/10.1002/jor.21172 (2010).

48. Lu, T. W. \& O’Connor, J. J. Bone position estimation from skin marker co-ordinates using global optimisation with joint constraints. J. Biomech. 32, 129-134. https://doi.org/10.1016/s0021-9290(98)00158-4 (1999).

\section{Acknowledgements}

This research was part-funded by CAMERA, the RCUK Centre for the Analysis of Motion, Entertainment Research and Applications, EP/M023281/1 and EP/T014865/1.

\section{Author contributions}

The work was conceived by L.N., M.E., D.C., L.W., P.M., S.C. L.N., M.E. \& L.W. collected the data. L.N. \& M.E. processed the data. L.N. analysed the data and prepared manuscript and figures. All authors (L.N., M.E., D.C., L.W., P.M., J.B., S.C.) revised and reviewed the manuscript. Funding was acquired by D.C. \& J.B. 


\section{Competing interests}

The authors declare no competing interests.

\section{Additional information}

Supplementary Information The online version contains supplementary material available at https://doi.org/ 10.1038/s41598-021-00212-x.

Correspondence and requests for materials should be addressed to L.N.

Reprints and permissions information is available at www.nature.com/reprints.

Publisher's note Springer Nature remains neutral with regard to jurisdictional claims in published maps and institutional affiliations.

(c) (1) Open Access This article is licensed under a Creative Commons Attribution 4.0 International License, which permits use, sharing, adaptation, distribution and reproduction in any medium or format, as long as you give appropriate credit to the original author(s) and the source, provide a link to the Creative Commons licence, and indicate if changes were made. The images or other third party material in this article are included in the article's Creative Commons licence, unless indicated otherwise in a credit line to the material. If material is not included in the article's Creative Commons licence and your intended use is not permitted by statutory regulation or exceeds the permitted use, you will need to obtain permission directly from the copyright holder. To view a copy of this licence, visit http://creativecommons.org/licenses/by/4.0/.

(C) The Author(s) 2021 